\title{
Do You Feel Like Passing Through Walls?: Effect of Self-Avatar Appearance on Facilitating Realistic Behavior in Virtual Environments
}

\author{
Nami Ogawa ${ }^{1}$, Takuji Narumi ${ }^{1,2}$, Hideaki Kuzuoka ${ }^{1,3}$, Michitaka Hirose ${ }^{1}$ \\ ${ }^{1}$ The University of Tokyo, ${ }^{2}$ JST PRESTO, ${ }^{3}$ University of Tsukuba \\ Tokyo, Japan \\ \{ogawa, narumi, kuzuoka, hirose\} @ cyber.t.u-tokyo.ac.jp
}

\begin{abstract}
Preventing users from walking through virtual boundaries (e.g., walls) is an important issue to be addressed in roomscale virtual environments (VEs), considering the safety and design limitations. Sensory feedback from wall collisions has been shown to be effective; however, it can disrupt the immersion. We assumed that a greater sense of presence would discourage users from walking through walls and conducted a two-factor between-subjects experiment $(\mathrm{N}=92)$ that controls the anthropomorphism (realistic or abstract) and visibility (full-body or hand-only) of self-avatars. We analyzed the participants' behaviors and the moment they first penetrated the wall in game-like VEs that gradually instigated participants to penetrate the walls. The results showed that the realistic full-body self-avatar was the most effective for discouraging the participants from penetrating the walls. Furthermore, the participants with lower presence tended to walk through the walls sooner. This study can contribute to applications that require realistic user responses in VEs.
\end{abstract}

\section{Author Keywords}

Self-avatar; Presence; Body Ownership

\section{ccS Concepts}

-Human-centered computing $\rightarrow$ Virtual reality;

\section{INTRODUCTION}

Proliferation of low-cost virtual reality (VR) devices and tracking systems has encouraged the development of room-scale applications that allow users to walk around in a virtual environment (VE). Compared with other locomotion modalities (e.g., teleportation and controllers), physical walking ensures natural and highly flexible movements [63]. However, the associated high degree of freedom also leads to issues when the users' movements need to be restricted within a certain

Permission to make digital or hard copies of all or part of this work for personal or classroom use is granted without fee provided that copies are not made or distributed for profit or commercial advantage and that copies bear this notice and the full citation on the first page. Copyrights for components of this work owned by others than the author(s) must be honored. Abstracting with credit is permitted. To copy otherwise, or republish, to post on servers or to redistribute to lists, requires prior specific permission and/or a fee. Request permissions from permissions@ acm.org.

CHI '20, April 25-30, 2020, Honolulu, HI, USA.

(C) 2020 Copyright is held by the owner/author(s). Publication rights licensed to ACM ACM ISBN 978-1-4503-6708-0/20/04 ...\$15.00.

DOI: https://doi.org/10.1145/3313831.3376562

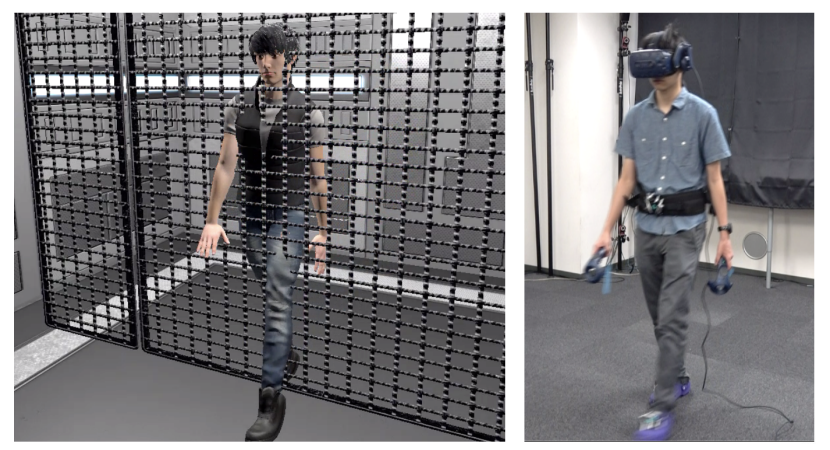

Figure 1. An avatar walking through a virtual wall (left) and the user in the real environment (right).

area or to follow certain paths. A prominent example is the problem of users walking through virtual boundaries, often represented by virtual walls, with which VR designers intend to confine the users within the boundaries (Figure 1). Conventional video games that typically use controllers simply restrict a virtual camera from passing through the walls. However, in room-scale VEs wherein users can freely walk around, the same strategy may cause a problem because the asynchronous movements of a user and a virtual camera can cause motionsickness. In addition, when the virtual walls represent the physical boundaries of the safety area, passing through these walls may lead to dangers such as hitting a real wall. Hence, it is necessary for room-scale VR applications to control the user's locomotion.

To address this problem, Boldt et al. [9] showed that a combination of auditory, visual, and vibrotactile feedback for head-wall and hand-wall collisions was effective in refraining players from walking through virtual walls without degrading the game experience. However, as this method can interfere with other vibrations or sound feedback that might be provided, especially during gaming, other methods that can discourage the users from passing through walls are required.

Our idea is to induce realistic user behavior, i.e., facilitating the users to instinctively avoid colliding with the wall. It is known that the users behave more realistically when they feel a higher sense of presence, the sense of "being there" in a VE [53, 48]. Therefore, we assume that a greater sense of 


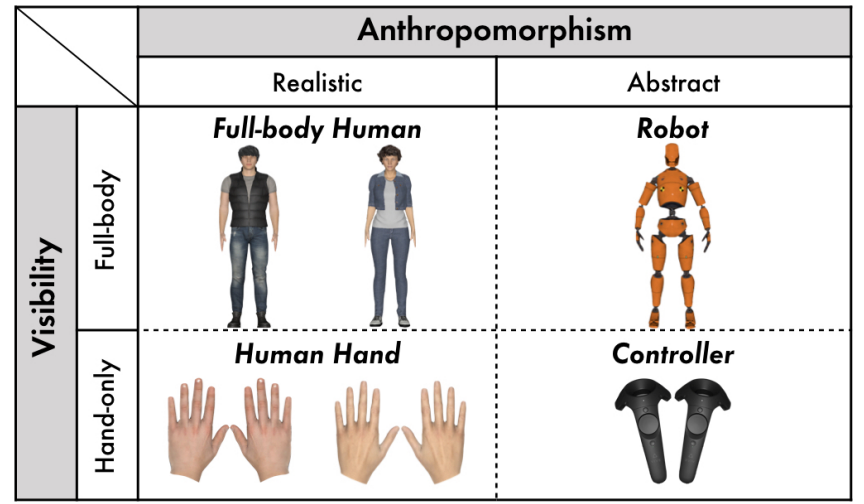

Figure 2. Four types of avatars were used in the experiment. For realistic avatars, gender-matching avatars were used (left: male, right: female).

presence would discourage users from walking through the virtual walls without providing them sensory feedback. In the presence research, the importance of the presentation of an avatar, i.e., a digital duplicate of one's body in VEs, has been pointed out [53]. A self-avatar is especially considered to enhance a form of presence called self-presence, i.e., the effect of embodiment on mental models of the self [6]. Moreover, recent studies have showed that the appearance of self-avatars, e.g., anthropomorphism (human-like - object-like) [49, 2, 30, 42] and visibility (full body - partial body) [61, 35], influences the presence and the sense of embodiment. Nonetheless, nonhuman-like (e.g., robots) or partial (e.g., hand and/or controller models) self-avatars are often used in current VR applications despite developments in VR technology. Hence, studies into how the visual fidelity of self-avatar affects users' behavior is important to the avatar and the presence research as well as in preventing users' penetration into the walls.

These backgrounds have motivated us to investigate how the anthropomorphism and visibility of a self-avatar affect the users' walk-through-wall behaviors in VEs with room-scale mapping. Thus, we conducted a between-subjects experiment where the participants $(\mathrm{N}=92)$ embodied one of the four self-avatars (Figure 2), which are combinations of anthropomorphism (realistic or abstract) and visibility (full-body or hand-only) factors. We hypothesized that the participants who used full-body and realistic self-avatars are more likely to behave in a VE as they would in a real environment (RE); this results in them being less likely to walk through walls as compared with participants who used unrealistic or partial self-avatars. Using a simple game-like VE that instigates the participants to walk through walls by giving them increasing incentives as they transit from room to room, we analyzed the tendency of the participants to walk through the walls. Additionally, we measured the presence physiologically (i.e., skin conductance response (SCR)) and subjectively (i.e., questionnaire). The behavioral data showed that the use of the full-body realistic avatar discouraged participants from walking through the walls. Furthermore, participants with a lower sense of presence tended to take less time to walk through the walls, despite no significant differences in the subjective ratings of presence and SCR among avatar conditions. These results provide quantitative evidence of the fact that a combination of high visibility and high anthropomorphism in self-avatars can induce realistic user behavior (i.e., avoiding the walls) in a VE, presumably owing to a higher sense of presence.

\section{RELATED WORK}

\section{Sensory Feedback of Virtual Walls}

Common feedback techniques used in current consumer VR games for avoiding collisions with virtual walls have been extensively reviewed by Boldt et al. [9]. According to them, the techniques strictly limit the game design and require additional strategies to alleviate the problem. Meanwhile, although it is uncommon in consumer VR games, providing haptic force feedback, e.g., wire-based force systems[40], pseudo-force feedback actuators[45], exoskeletons[21], and electrical muscle stimulation[32], can be effective in physically preventing users' arms and hands from penetrating the wall. In addition, passive haptics, which use physical objects to provide haptic feedback from virtual objects, can also be effective. Specifically, hand retargeting techniques, wherein the movements of a virtual hand are mapped to guide the user's actual hand toward physical haptic props for providing passive haptics for multiple virtual objects [4, 12], can be effectively used to prevent a virtual hand from penetrating virtual objects [11, 70]. However, while these haptic feedback techniques can be implemented for hand penetration, they do not address the problems with the head, passing through the walls, which determines the viewpoint in immersive VR. Generally, mapping a virtual viewpoint that is different from the actual head movement could induce motion sickness, although it has been shown that humans can tolerate a small amount of rotational shift in the viewpoint [57]. Furthermore, even though there has been an attempt to provide passive haptics such as walls without redirecting viewpoints, it requires several volunteers to carry physical props in real time [13].

Although existing studies have focused primarily on haptic feedback systems in general, Boldt et al. [9] investigated the effect of other sensory feedback on preventing users from walking through walls. They proposed a combination of visual, auditory, and vibrotactile feedback for both hand-wall collisions (vibrating controllers and presenting a knocking sound) and head-wall collisions (blackened vision and dampened surrounding sound), which is non-intrusive and requires no special equipment. They confirmed that users who received the feedback were less likely to walk through walls than other users. Nevertheless, contrary to all aforementioned approaches, which provide sensory feedback from wall collisions, our approach aims to prompt realistic user behavior, that is, avoiding collision with the walls by inducing a higher sense of presence without any feedback or instruction.

\section{Effect of Self-avatar on Perception, Action and Behavior}

The importance of a self-avatars has long been established in the presence research [53]. This means that the self-avatars can exert an impact on users' behavior in VEs because a sign of presence is presumed to be evoked if participants within a VE behave as if they were in an equivalent RE [48]. Indeed, presence is operationally defined by such "respond as if real" behaviors, where response is considered at multiple levels: 
subjectively (e.g., by questionnaire [64, 66]), behaviorally (e.g., through looming responses [22], postural sways [17], and aftereffects [55]), and physiologically (e.g., by heart-rate [38] and SCR measurements [14]). However, while the effect of the presentation of a self-avatar (vs. without self-avatar) and the visual fidelity of avatars of others on presence has been well investigated, especially in communication research [53, $5,41]$, few studies have focused on how visual fidelity of selfavatars influences presence, showing only the inconclusive evidence [49, 29, 65, 25].

In contrast, the effect of visual fidelity of self-avatars on the sense of body ownership (SoBO) has been extensively investigated. SoBO refers to the sense of one's self-attribution of a body [26], and it is a concept analogous to self-presence. Fundamentally, the closer the appearance of a self-avatar is to one's own body, in terms of structural and morphological aspects, the stronger the SoBO [27, 26]. For instance, a realistic virtual human hand elicits a stronger SoBO than nonanthropomorphic hands (e.g., robotic or cartoon) [2, 30, 50] and objects (e.g., sphere, board, block, and arrow) [2, 42, 30, 69]. In addition, the connectivity of hands with a torso (i.e., the visibility of arms) has been considered one of the important factors for evoking SoBO $[60,61,35]$ although, to the best of the authors' knowledge, no studies have compared hand-only and full-body self-avatar representations. As in the case of presence, "respond as if real" is also a common indicator of the strength of SoBO. Typically, this response is measured based on the behavior or physiology of the user when an avatar is exposed to a virtual threat (e.g., a falling object $[69,20]$, fire [2, 34, 58], or a saw [28]). Behavioral measurements have shown that participants experiencing SoBO are more likely to avoid virtual threats $[20,28,58]$. Considering these studies on presence and SoBO, we can assume that the higher the visual fidelity of a self-avatar (i.e., the closer the appearance is to our own body), the stronger the sense of presence as well as SoBO, facilitating the user's more realistic behavior in a VE.

Other than the evidence from studies on presence and SoBO, existing research also recognizes the critical role of self-avatars on a wide range of human information processing in VEs. For example, egocentric distance estimation becomes more accurate when a self-avatar is displayed compared with when no-avatar is displayed [46]. In addition, a decrease in avatar visibility (e.g., full body, only joint locations, or end-effectors) impairs distance estimations for both near-field [15] and farfield distances [47]. Furthermore, a recent study has suggested that anthropomorphism also affects object size perception when the virtual hand sizes differ from actual sizes [42].

In contrast to the effect of a self-avatar on a user's perception, the effect on the actions and behavior appears to be complex. For example, a self-avatar, compared with when no-avatar is displayed, improved the cognitive task performance [56]. The presentation of a self-avatar also improved the performance of locomotion and object interaction tasks [37], but did not influence these tasks when the field of view is limited [59]. In addition, it has also been shown that connectivity of hands with a torso (i.e., arm visibility) does not influence the performance of object selection tasks [62]. Furthermore, another study demonstrated that the anthropomorphism of a full-body avatar had a significant effect on the accuracy of pointing [51]. Recently, Lugrin et al. [33] compared three types of self-avatar representations (controller, hand, or upper body) on players' experience and performance in an action-based VR game. Their results did not reveal any significant differences in the perceived control and difficulty of the game, as well as SoBO, immersion, and involvements.

Several studies have investigated the effect of self-avatars on user's behavior with respect to affordance judgment, i.e., deciding what they can and cannot do in the environment. Lin et al. [31] showed that the presentation of self-avatars induced a judgment similar to that in a RE when stepping off a ledge and stepping over or ducking under a pole in a VE. Bodenheimer et al. [8] further compared situations involving no self-avatar, a line-based skeleton avatar, and a realistic human avatar using a virtual ledge. Their results replicated the work of Lin et al. [31]; however, they found no effect of anthropomorphism. In contrast, Alshaer et al. [1] showed that the presentation of a self-avatar did not influence whether a participant passed through or went around a particular gap, even though it affected the sense of presence. Altogether, anthropomorphism and visibility are important attributes of self-avatars, potentially influencing user behavior. However, the effects of these factors are highly complex and task-dependent. Thus, further investigation is required to reveal the effects on behavior, with regard to walking through walls.

\section{EXPERIMENT}

A between-subjects experiment was performed to investigate whether the anthropomorphism (realistic or abstract) and visibility (full-body or hand-only) of self-avatars discourage participants from walking through the walls. The participants were instigated to walk through the virtual walls in a VE with room-scale mapping, while embodied with any of four types of self-avatars. We analyzed the participants' behavior using motion-tracking data with two main indices: whether (i.e., the number of participants) and when (i.e., duration) they walked through the walls. Additionally, we analyzed their physiological reaction to the virtual threats using SCR as an indicator of presence and SoBO, as well as the subjective scores of presence and $\mathrm{SoBO}$ using a questionnaire.

\section{Participants, Apparatus, and Avatar Appearance}

We conducted the experiment with 92 participants (64 males and 28 females, $30.48 \pm 9.29$ (SD) years old), recruited from the public through social media. Only a summarized aim of the experiment (i.e., to evaluate user experiences in room-scale VR) was announced when recruiting the participants. These naive participants were assigned to any avatar condition to ensure that each group had the maximum similar distribution of gender and prior experience with VR. Thirty participants had no previous experience with VR, forty-four had few previous experiences, eight occasionally used VR, eight often used VR, and two used VR daily. They signed an approved statement of consent, and they were compensated with an Amazon gift card amounting to approximately $\$ 5$. The experiment was approved by the local ethical committee and conducted at a room at the university. 

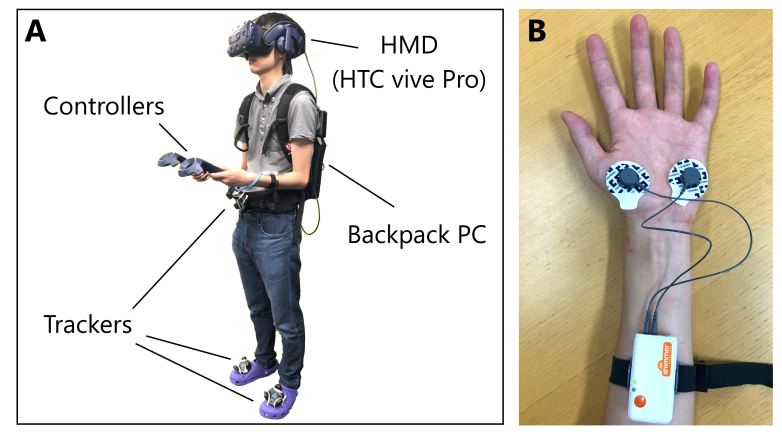

Figure 3. (A) A participant equipped with experimental apparatus. (B) A wearable sensor used to estimate participants' SCR to threat.

The experimental apparatus included an HMD (Vive Pro, HTC), three position trackers (Vive Tracker, HTC), a pair of controllers (Vive Controller, HTC), a wearable SCR sensor (Shimmer3 GSR+ unit, Shimmer), and a Windows-based backpack computer (VR One 6RE-002JP, MSI) (Figure 3A). Two trackers were attached to the top of the shoes and a tracker was attached to the belt on the waist. The HMD, trackers, and controllers were tracked using HTC's Lighthouse system. The controllers were only used to track the positions of the users' hands; the buttons were not used. To display the VEs, we used the backpack computer with an Intel i7-6820HK, 32 GB RAM, and NVIDIA GTX1070 to ensure that the participants could freely walk around without being bothered by the cables. The experimental program was developed using Unity $3 \mathrm{D}$ and ran at a frame rate of $100 \mathrm{~Hz}$ on average.

We used four types of avatar appearances with different levels of anthropomorphism and visibility, as shown in Figure 2. We used Full-body Human: realistic full-body 3D models obtained from Morph 3D, Robot: a robotic humanoid avatar created by CGBoat, Human Hand: the hand parts of the avatars used in the Full-body Human condition, and Controller: a controller model that was obtained from HTC. For the realistic avatars, we used male and female gender-matching avatars. For the full-body avatars, the full-body animation was calculated via inverse kinematics using the VRIK package. The avatar's height was set to the participants' height. In addition, at the beginning of the experiment, the participants looked at a virtual mirror in the scene so that they would see a reflection of their virtual body. This procedure is often used to enhance SoBO, particularly when using full-body avatars [54, 44].

\section{Skin Conductance Response (SCR)}

SCR, a widely accepted indicator of autonomic arousal, has been shown to be a reliable physiological index of presence and SoBO $[14,3,16]$. We provided the participants with virtual threatening stimuli and measured their physiological response to the stimuli using SCR. SCR is considered to reflect both $\mathrm{SoBO}$ and presence because a higher SoBO and presence would both contribute towards a realistic reaction to the threat in the VE. To measure SCR, two electrodes were attached to the left palms of the participants using Kendall Arbo H135 (COVIDIEN) (Figure 3B). The galvanic skin response (GSR; the variation of the electrical conductance of the skin) signals were recorded and analyzed using Tobii Pro Lab (Tobii Tech- nology K.K.). We used the event-related SCR, defined as the amplitude of the peak in GSR that occurred $1-5 \mathrm{~s}$ after the onset of the threatening stimuli, as an index of SCR analysis.

\section{Experimental Scene}

We followed the experimental setup of Boldt's study [9]. We only slightly modified the design to ensure that it could be implemented in line with our study. There were four virtual rooms $(4 \times 4 \mathrm{~m})$ that provided the participants with different levels of incentives to walk through the walls (Figure 4). In each room, the participants were asked to move from the starting point to the goal where the teleporter to the next room was located. They were tasked with pushing all buttons in the correct order to activate the teleporter. As stated in [9], most users rarely attempt to walk through walls in brief play sessions unless there is a clear incentive. Therefore, we designed virtual rooms to quickly instigate the participants to walk through the walls as follows; (1) The inner walls were made of 5-cm-thick chain link fence instead of solid walls so that the participants could easily see the buttons over the wall. (2) No feedback was provided on head- and hand-collision with walls, and hands or controllers could penetrate the walls. However, visual and auditory feedbacks were given when the button was pressed or the teleporter was activated. To avoid any influence of the ease in pushing buttons, the collision-detection areas of the avatar models, which were used to determine whether the button was pressed, were the same for all avatars. (3) A time limit was set for each room. The participants saw a timer counting down from $150 \mathrm{~s}$. In room 3, the time was exceptionally set for $180 \mathrm{~s}$. If the time was up, the teleporter was activated spontaneously.

Room 1 was designed to instigate the participants to penetrate their hand into the wall. Three pairs of two successive buttons were placed next to each other across the wall. Hence, if the participants penetrated their hand, they could easily push the paired buttons without walking around. Alternatively, the participants could walk around the walls or insert their hand through the aperture in the wall to push the button nearby. Thus, the incentive to penetrate the body was thus kept little to avoid too many participants penetrating in the first room and moderately control the incentives of penetration throughout the entire experiment.

Room 2 provided an incentive to the participants to walk through the wall to solve a repetitive and time-consuming task faster (Figure 4). A total of twenty buttons were used. As odds and evens were separately located across the wall, the participants must walk back and forth between two locations that were spatially close but separated by the wall. The timelimit was set so that they could successfully complete in time when they walked fast. However, if they walked through the wall, they could easily push the buttons from the other side of the wall without walking around it. The buttons right next to the wall $(2,9,12$, and 19) could be pushed without head-wall collision if they inserted their arm.

Room 3 was impossible to complete without walking through a wall. It had three buttons. Buttons 1 and 3 were located at the area surrounded by the two sliding walls, which closed when the participants got closer; thus, the buttons could be pushed only if the participants walked through the walls (Figure 4). 


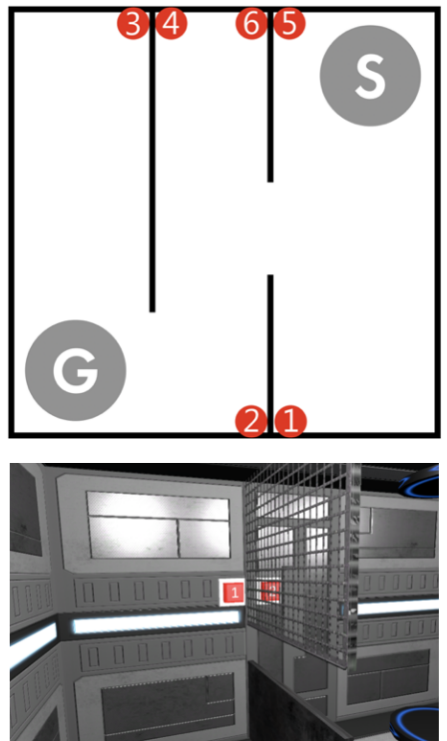

Room 1

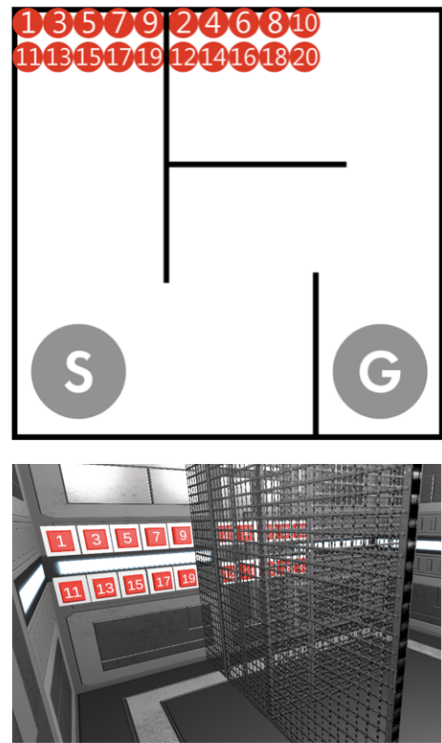

Room 2

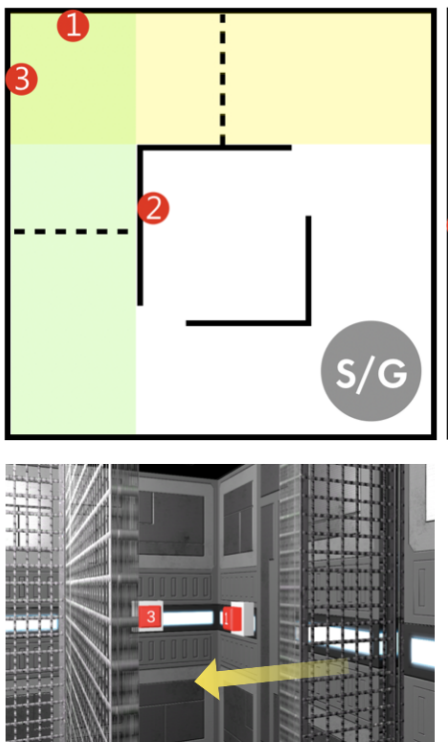

Room 3
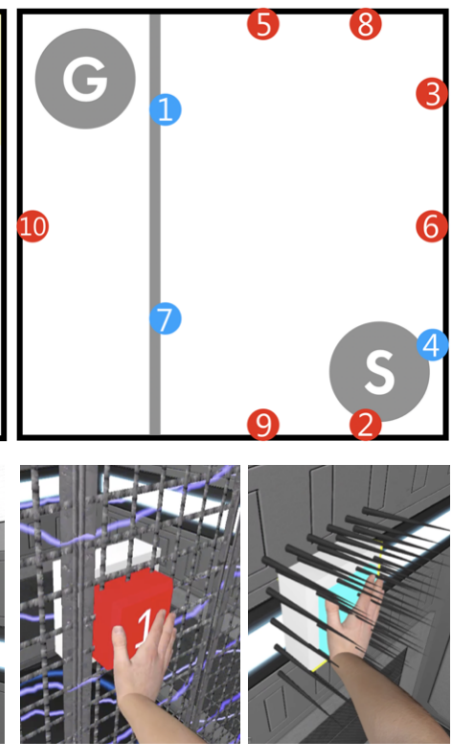

Room 4

Figure 4. (Top) Room layouts. S and G represent the start and goal, respectively. The lines represent the walls. The buttons are numbered and must be pushed sequentially to activate the teleporter at the goal in each room. The dashed lines in room 3 represent the sliding walls that closed when the participants entered the yellow- and green-colored areas. The gray line in room 4 represents the wall through which electric lightning was passed when the participants approached it. On pushing the blue buttons in room 4, the participants were presented with the threatening stimuli. (Bottom) Room characteristics. In Room 1, the participants could easily push the button on the other side of the wall if they penetrated their hand. However, they also had the option to pass their hand under the wall and walk around. In Room 2, the participants had to walk back and forth several times but could take a shortcut if they walked through the wall. In Room 3, the buttons could only be pushed if the participants walked through the sliding wall, which closed when they approached. In Room 4, threatening stimuli such as electric lightning passing throughout the wall (left) and a bunch of needles popping out from the wall (right) were presented.

We expected remarkable differences in the time until the participants first walked through the wall rather than whether or not they did so; this is because it would become obvious that they had no choice than walking through the wall to push the buttons immediately after they tried several other possible solutions. Hence, the time-limit was set to $180 \mathrm{~s}$, which is 30 $\mathrm{s}$ longer than in the other rooms; this was done to allow sufficient variation in time to reflect the participants' reluctance to walk through the walls.

Room 4 was also impossible to complete without walking through the wall and it was more obvious than room 3 . Nevertheless, the main purpose of room 4 was to provide the participants with threatening stimuli to measure SCR. There were a total of ten buttons. Buttons 1 and 7 were located on the wall and if a participant's hand got closer to the wall, electric lightning visually and auditorily passed through the wall. In addition, when button 4 was pushed, a number of needles popped out from the wall. Furthermore, they had to walk through the wall, which electrically lit up when they got closer, to push button 10, which was located on the other side of the wall. We expected the participants who felt a greater presence or SoBO to exhibit the greater SCR and to hesitate before walking through the lightning wall.

\section{Questionnaire}

The subjective evaluation of $\mathrm{SoBO}$ and presence was assessed using Gonzalez-Franco and Peck's avatar embodiment questionnaire [18] and the Slater-Usoh-Steed questionnaire [64], respectively (see Appendix Table 1 for items). Among the six subsets that constitute the avatar embodiment questionnaire [18], we used the questions about 'Response to external stimuli' as well as 'Body ownership', as several experiments have shown correlations between the questionnaires and the physiological and behavioral responses for bodily threats [36, 19]. Hence, we used the 'Response to Threat' scale as the index reflecting both SoBO and presence, similar to the case of SCR. All questionnaire items were supplemented by a Japanese translation. Each response was scored on a sevenpoint Likert scale $(-3=$ strongly disagree and $+3=$ strongly agree). In addition, we asked the participants to rate their VRand video-game-experiences on a scale of 1 to 5 to test if these characteristics influenced their behaviors.

\section{Procedure}

Upon entering the room, the participants read and signed an experiment consent form. Thereafter, the experimenter set the SCR sensor on the participants' left hand. Next, the participants put on the belt and the shoes with trackers. After wearing the backpack computer and the HMD, they were given controllers and instructed not to grip the controller strongly because muscular activity could introduce noise in measuring the SCR. They were initially located in the virtual room without any walls for instruction of the rules. They were instructed not to go outside the exterior walls for their safety. Thereafter, the virtual mirror appeared in front of them. They were asked to look at the mirror while freely moving their bodies for $30 \mathrm{~s}$. They were then asked to walk around the room while looking down at their lower bodies. These processes were aimed to 
ensure the participants' embodiment of their avatar. Next, they were instructed on how to push the buttons, use the teleporters, complete their tasks, and that there were four rooms with a certain time limit. They were told that if they mistakenly pushed the button with incorrect number, they had to start over from the first one. They were also instructed to head for the teleporter if they exceeded the time-limit. Nevertheless, they were neither informed about the walls, nor did they see the walls inside a room before entering room 1. Before entering room 1, they were told that they could not ask any questions during the experiment. When they went to the activated teleporter in each room, they were automatically transported to the next room and the timer started to count-down. If some participants did not walk through any wall even after $30 \mathrm{~s}$ had passed beyond the time limit of the last room, the experimenter told them to walk through the wall and complete the task. This procedure was introduced to ensure the SCR against the lightning wall could be measured for all the participants including those who did not walk through the wall in time, and the value of $30 \mathrm{~s}$ was determined through the preliminary experiment. In the actual experiment, only one participant was instructed to do so. After removing all the apparatus, the participants answered the questionnaire regarding SoBO, response to threat, presence, and demographics through a web form. Finally, the experimenter conducted a semi-structured interview with the participants on why they decided to walk through the wall and how they felt during the VR experience. After the interview, the experimenter debriefed the true purpose and hypotheses of the experiment when asked by the participant.

\section{Hypotheses}

[H1] The more realistic and visible the self-avatars, the greater number of participants refrain from passing through the walls.

[H2] The more realistic and visible the self-avatars, the longer the participants take time before passing through the walls.

[H3] The more realistic and visible the self-avatars, the stronger the SoBO and presence.

[H4] The participants with a higher sense of presence tended to refrain from passing through the walls.

\section{RESULTS}

\section{Behavioral Data}

For the behavioral analysis, we followed the analysis method used in Boldt et al.'s study [9] but revised it according to our study design. For the behavioral data, we analyzed the number of participants who walked through the walls ([H1]) and the time until they first walked through ([H2]) for each room based on the head tracking data. As ANOVA is known to be robust to normality assumption, we conducted ANOVA for continuous data even when the data were not normally distributed. Two-way factorial ANOVA was used considering the between-group factors of anthropomorphism (two levels: realistic and abstract) and visibility (two levels: full-body and hand-only). Controller, Human Hand, Robot, and Full-body Human conditions are denoted by C, H, R, and F, respectively, for readability. For the distribution of the participants' behavior in each room, we provided the stacked bar chart with percentages in Figure 5. (See Table 2 in Appendix for the combinations of raw numbers and percentage.)

\section{Room 1}

In Room 1, we did not expect the participants to walk through the walls except to insert their hands. Nevertheless, a few participants (C: 1, H: 2, R: 1, F: 0) walked through. The participants' other typical behavior to push the nearby button obstructed by the wall was classified as follows: to penetrate their hand into the wall (C: 2, H: 4, R: 2, F: 1), to pass their hand through the aperture in the wall (C: 3, H: 2, R: 2, F: 2), and to make a detour (C: 17, H: 15, R: 18, F: 20) (Figure 5) Although we counted the passing-hand and detour groups separately, we were interested in the ratio of the participants who penetrated their hands into the wall, including those who walked through the walls. To test if the ratio was influenced by each between-group factor (anthropomorphism and visibility) and their interaction, we used a binary logistic regression. The likelihood ratio test showed that no variables significantly explained the ratio (anthropomorphism: $\chi^{2}(1)=0.09, p=.76$, visibility: $\chi^{2}(1)=2.29, p=.13$, interaction: $\chi^{2}(1)=2.31$, $p=.13)$. Hence, contrary to [H1], the number of participants who penetrated their hand into the wall was not statistically different depending on their avatars.

\section{Room 2}

In Room 2, some participants (C: 7, H: 13, R: 8, F: 5) walked through the walls to take a shortcut as expected (Figure 6 left and Figure 5). Nonetheless, several participants (C: 5, H: 3, R: 6, F: 2) penetrated their hands into the wall (Figure 6 center and Figure 5). Although only a few participants (C: 1, H: $0, \mathrm{R}: 1, \mathrm{~F}: 1$ ) did so to touch the button, most of them typically withdrew their hand soon after penetration on the
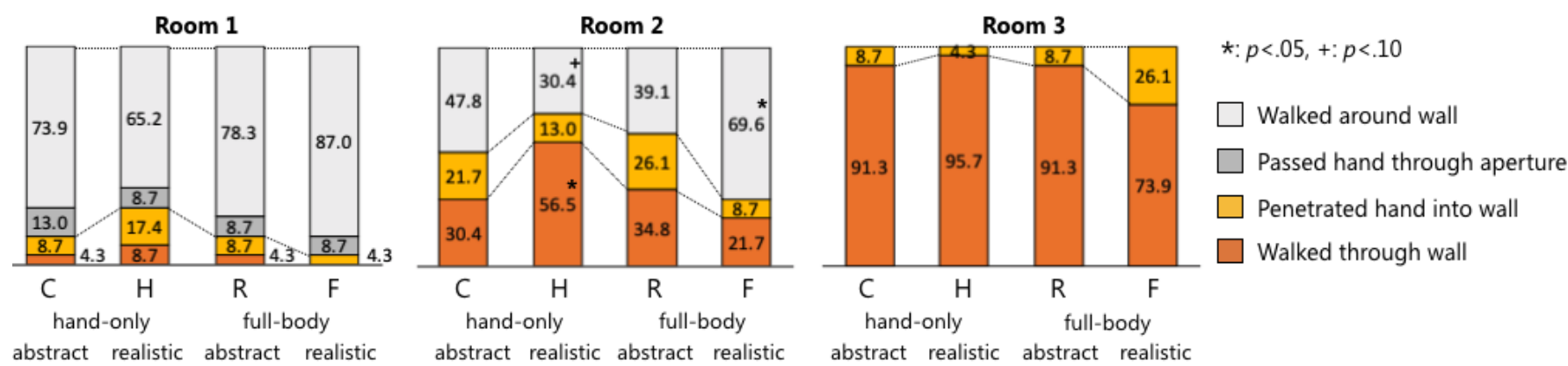

Figure 5. Distribution of participants' behavior in rooms 1, 2, and 3 according to avatar anthropomorphism and visibility. C, H, R, and F represent Controller, Human Hand, Robot, and Full-body Human, respectively. The shown numbers are the percentage out of 23 participants for each condition. 
wall away from the buttons. According to the semi-structured interviews, 11 out of 16 participants intentionally did that to check if they could or were allowed to put their hand beyond the wall, and they determined that they were allowed to pass through the wall because no feedback (e.g., visual or haptic) or penalty (e.g., alert screen or audio) occurred. This "confirmation behavior" was also often observed in the participants who eventually walked through the wall (C: 4, H: 3, R: 3, F: 2). Conversely, the remaining participants who walked through the wall (C: 3, H: 10, R: 5, F: 3) walked through the wall straight without any confirmation behavior. Eventually, the remaining participants (C: 11, H: 7, R: 9, F: 16) walked the long way around the wall without contacting the wall to the end (Figure 6 right and Figure 5).

To test if the distributions of the three types of participant behavior were influenced by avatars, we conducted the likelihood ratio test of the ordered logistic regression model. The result revealed that the interaction effect was significant $\left(\chi^{2}(1)=6.06, p<.05\right)$. A post-hoc adjusted residual analysis of a Chi-squared test showed that the number of participants who walked through the wall in the $\mathrm{H}$ condition and the number of participants who did not contact the wall in the F condition were significantly larger than the corresponding expected values (residual $=2.38, p<.05$; residual $=2.53, p<.05$ ). Each expected value was calculated under the null hypothesis that the self-avatars and behaviors were independent. Additionally, the number of participants who did not contact the wall in the $\mathrm{H}$ condition demonstrated a trend of being smaller than the expected value (residual $=-1.81, p=.07$ ). These results supported [H1].

\section{Room 3}

In room 3, most participants walked through the wall (C: 21 , H: 22, R: 21, F: 17; Figure 5). The likelihood ratio test of the binary logistic regression model showed that no variables significantly explained the ratio of the participants who walked through the wall against who did not so (anthropomorphism: $\chi^{2}(1)=0.97, p=.33$, visibility: $\chi^{2}(1)=2.69, p=.10$, interaction: $\left.\chi^{2}(1)=1.91, p=.17\right)$. Although we expected that the ratio to be different depending on avatar groups ([H1]), the result was not surprising considering that Room 3 could not be solved obviously without entering the walls.

However, it was typical that they had been looking for the gimmick to open the door before they entered the wall, al-
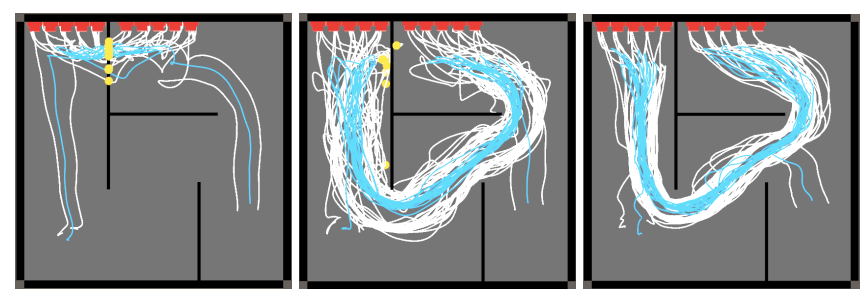

Figure 6. Representative trajectories for typical behavior patterns in room 2. (left) A participant who walked through the wall. (center) A participant who penetrated his/her hand into the wall but never walked through the wall. (right) A participant who did not contact the wall at all. The trajectories of head and hands are colored in white and blue, respectively. Yellow dots represent collisions of head or hands with walls. though a part of the participants (C: 11, H: 13, R: 11, F: 6) walked through the wall straight without hesitation. Hence, even though the binary counting indicated a ceiling effect and did not support [H1], the time until the participants first walked through the wall in the room largely varied. Thus, we next analyzed the time data among the participants who walked through the wall to test [H2]. The time data were not normally distributed. Two-way factorial ANOVA was conducted (Figure 7 left). The result showed a significant interaction effect $\left(F(1,77)=6.44, p<.05, \eta_{p}^{2}=0.08\right)$. Finally, Tukey's post-hoc tests $(\alpha=.05)$ were conducted for pairwise comparisons. The result demonstrated that the time in the $\mathrm{F}$ condition was longer than that in the $\mathrm{H}$ and $\mathrm{R}$ conditions $(\mathrm{H}$ : $t(77)=2.64, p<.05$, R: $t(77)=2.44, p<.05)$. Although we did not find any significant difference in the ratio, the ratio data and time analysis exhibited a similar tendency that the participants under the $\mathrm{F}$ condition hesitated in walking through the wall. Note that the time analysis did not include the data from the participants who did not walk through the wall. These results supported [H2].

\section{Room 4}

In room 4, all participants walked through the wall in the end. However, a few of them (C: 1, H: 1, R: 0, F: 2) passed after the time was up. Only one participant, under the F condition, was instructed by the experimenter to walk through the wall in accordance with the rule as she did not walk through even though 30s had passed after the time-limit. Some participants seemed to hesitate to walk through the lightning wall, including the participants who had already walked through the walls before room 4 . Figure 8 is the visualization of the average velocity of head movement over time in the temporal proximity of the wall penetration for each avatar type. The velocity was calculated at each frame and then averaged among each time window of $0.1 \mathrm{~s}$ for each participant. By setting the moment of the wall penetration to time 0 , the time series data of velocities were averaged among the participants. For those $(\mathrm{N}=15)$ who passed through the walls more than once, we averaged the velocities regarding all the wall penetrations for each participant. Although we did not conduct any statistical test on the velocity data, several interesting characteristics can be observed from Figure 8. First, the participants tended to speed up while penetrating the wall irrespective of avatar types. Second, the participants in the F condition seemed to move more slowly than others before penetrating the wall, indicating that they might have hesitated to penetrate it than the others. Qualitative observations of the behavior also indicate that some participants stepped back before walking through the wall, whereas a few participants, especially those who had not walked through the wall before, took time to search for the gimmick, similar to room 3 . These characteristic behaviors remarkably appeared in the time until the participants walked through the lightning wall after they pushed button 9 . Thus, the time can be considered to reflect the amount of psychological resistance to walk through the lightning wall. It can also be considered to reflect presence in the sense that the resistance is a realistic response to a threat. Moreover, time data were not normally distributed. Two-way factorial ANOVA showed a significant interaction effect $\left(F(1,88)=9.88, p<.01, \eta_{p}^{2}=0.10\right)$ (Figure 7 center). 

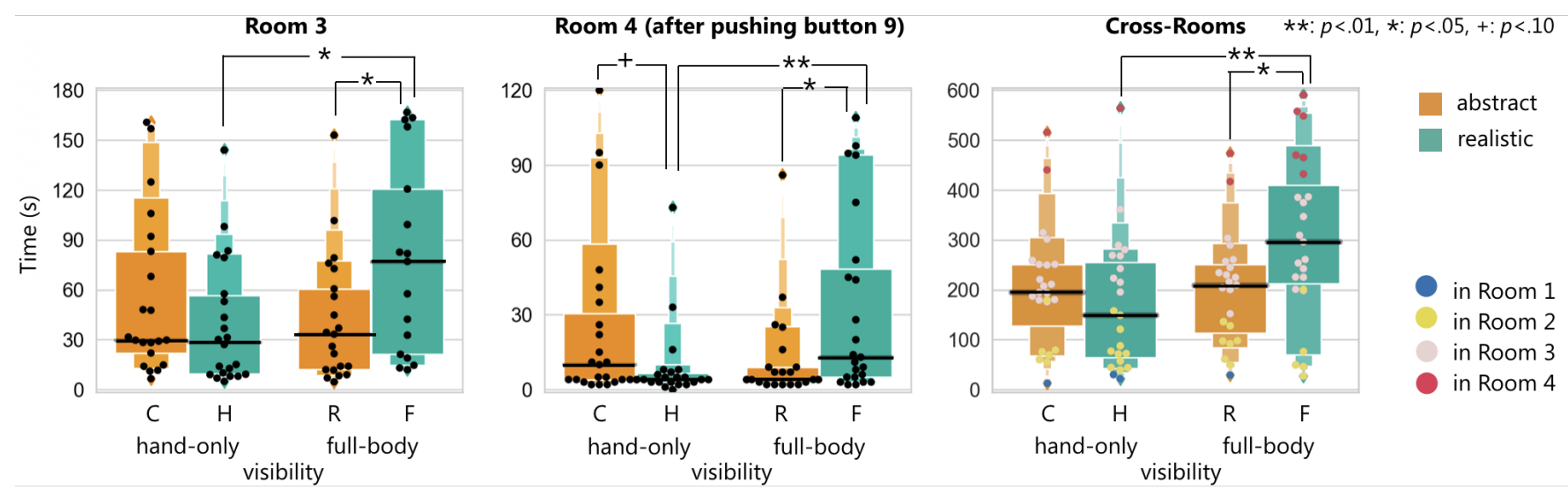

Figure 7. Letter-value box plot ${ }^{1}$ with data points of the time until the participants first walked through the wall in room 3 (left), room 4 (center), and across rooms (right) according to avatar anthropomorphism and visibility. C, H, R, and F represent Controller, Human Hand, Robot, and Full-body Human, respectively. In the cross-room results, the data points are colored according to the rooms where the participants first walked through the wall.

Tukey's post-hoc tests $(\alpha=.05)$ showed that the time in the $\mathrm{F}$ condition was longer than that in the $\mathrm{H}$ and $\mathrm{R}$ conditions $(\mathrm{H}$ : $t(88)=2.88, p<.01, \mathrm{R}: t(88)=2.53, p<.05)$. In addition, the time in the $\mathrm{C}$ condition showed a trend to be longer than that in the $\mathrm{H}$ condition $(t(88)=1.92, p=.06)$. These results indirectly supported [H3].

\section{Cross-rooms results}

To determine whether the participants' behavior throughout the experiment was influenced by self-avatars, we calculated the total time spent by each participant before they first walked through the wall, from the start time of the experiment. The time data were normally distributed except for the $\mathrm{H}$ condition. Two-way factorial ANOVA showed a significant interaction effect $\left(F(1,88)=6.09, p<.05, \eta_{p}^{2}=0.06\right)$ (Figure 7 right). Tukey's post-hoc tests $(\alpha=.05)$ revealed that the time under the $\mathrm{F}$ condition was longer than the time under the $\mathrm{H}$ and $\mathrm{R}$ conditions $(\mathrm{H}: t(88)=3.41, p<.001, \mathrm{R}: t(88)=2.60$, $p<.05)$. These results supported [H2].

\section{Physiological Data: Skin Conductance Response}

As a physiological measurement of presence and SoBO, SCRs for the four threatening stimuli in room 4 were analyzed to

\footnotetext{
${ }^{1}$ By plotting more quantiles than in a conventional box plot, a lettervalue box plot provides additional information about the shape of the distribution, particularly in the tails [23].
}

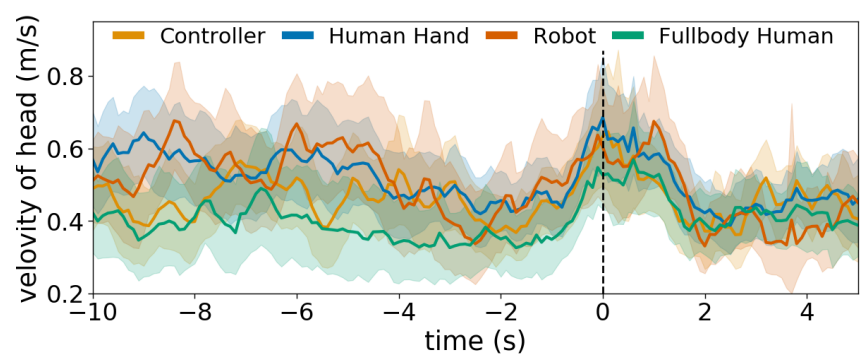

Figure 8. Line plot of the velocity of head movement over time in the temporal proximity of wall penetration in Room 4 for each avatar type. Time 0 indicates the moment the participants penetrated the wall. Translucent bands indicate $95 \%$ CIs, estimated by 1,000 bootstraps. test [H3]. The data for some participants (C: 4, H: 1, R: 4, $\mathrm{F}: 2$ ) were not recorded due to technical issues. Hence, the following analysis was conducted excluding those data. In addition, if no peak in GSR was detected 1 to 5 s after the threat, the SCR was calculated as 0 . The data was normalized using the Log of SCR magnitude +1 and standardized by Z-scores using mean and standard deviation of GSR metrics 0 to $5 \mathrm{~s}$ before each threat due to the GSR signal characteristics in accordance with [10]. For the time series graph of mean GSRs for each threat, please see Appendix Figure 1. Among the SCRs of the four threats, the threat of walking through the lightning wall affected the whole-body whereas the others worked for hand. Therefore, we analyzed these separately. We calculated the mean SCRs among the first three threats (button 1, 4, and 7) as hand-threat and the SCRs of the last threat (walking through the lightning wall) as full-bodythreat. For hand-threat, two-way ANOVA showed no significant effects (anthropomorphism: $F(1,77)=0.20, p=.66$, $\eta_{p}^{2}<0.003$, visibility: $F(1,77)=1.63, p=.21, \eta_{p}^{2}=0.02$, interaction: $\left.F(1,77)=0.73, p=.40, \eta_{p}^{2}=0.009\right)$. For fullbody-threat, two-way ANOVA only showed a trend of main

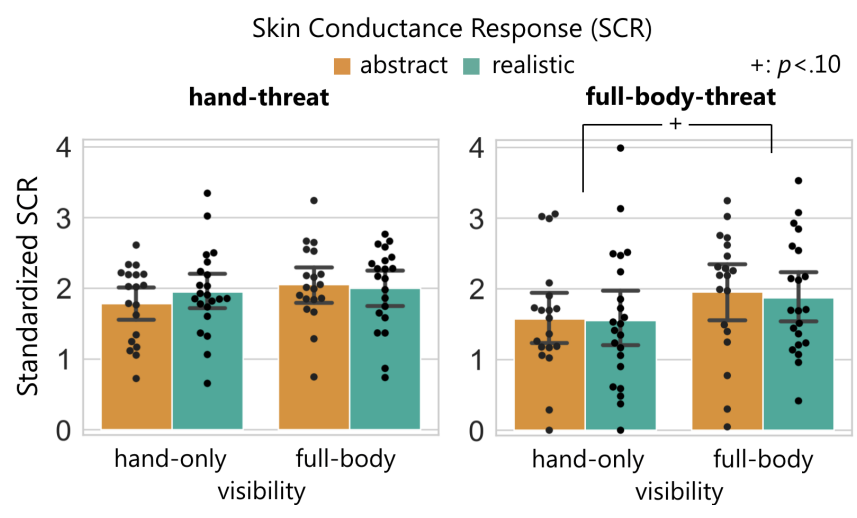

Figure 9. Bar plots with data points of SCR for the hand-threats and full-body-threat in room 4 according to avatar anthropomorphism and visibility. The SCR for hand-threats is the mean SCR for the three handthreats. Error Bars indicate $95 \%$ CIs, estimated by 1,000 bootstraps. 

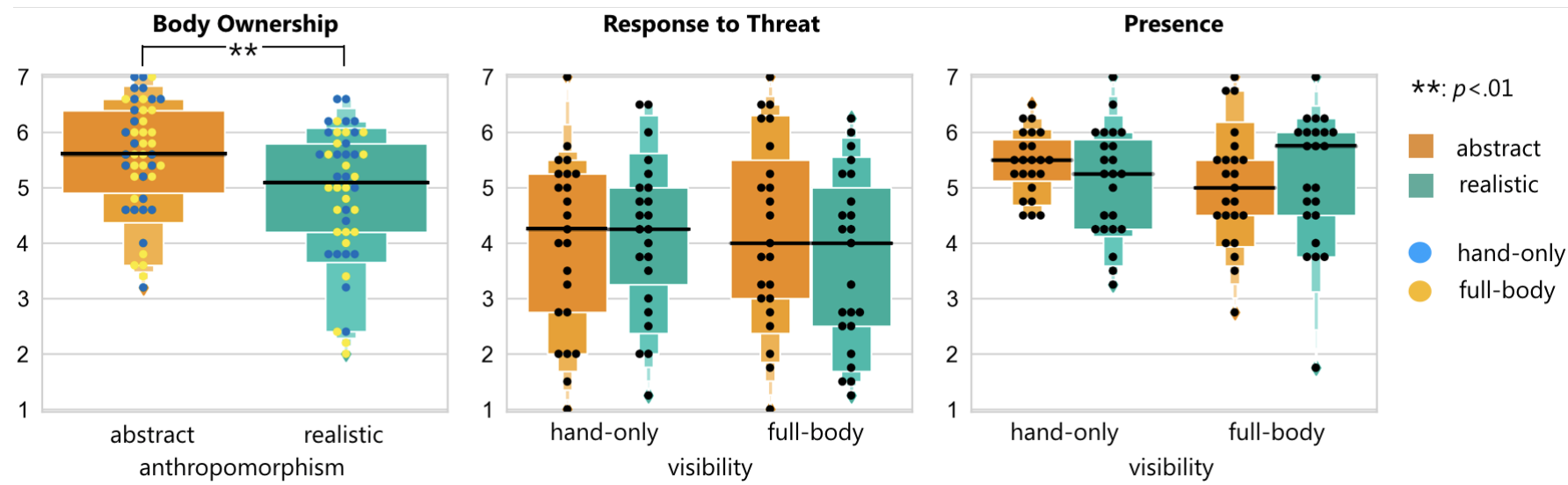

Figure 10. Letter-value box plots ${ }^{1}$ with data points of the subjective ratings (from -3 to +3 ) of Body ownership (left), Response to threat (center), and Presence (right) obtained through the questionnaires according to avatar anthropomorphism and visibility.

effect of visibility $\left(F(1,77)=3.27, p=.08, \eta_{p}^{2}=0.04\right)$; the SCR for full-body-threat under the full-body condition tended to be greater than that under the hand-only condition (Figure 9). These results did not support [H3].

\section{Subjective Data: Questionnaire}

To further test [H3], the subjective ratings of the questionnaire were analyzed. For each scale (Ownership, Response, Presence), we first checked internal consistency. Because some items of Presence (P4 and P6) were negatively correlated with other items, we excluded these items. Consequently, Cronbach's $\alpha$ s were $\alpha=0.69$ (Ownership), $\alpha=0.8$ (Response), and $\alpha=0.56$ (Presence). The answers for each item were aggregated and averaged (answers for control items were inverted) to compute the scores for each scale per participant. Because Likert scale is considered to be an ordinal scale, an aligned-rank transform, which allowed the use of ANOVA to analyze the interaction effects with the non-parametric data [67], was first applied and then two-way ANOVA was conducted (Figure 10). For Ownership, the ANOVA revealed a significant main effect of anthropomorphism $(F(1,88)=8.63$, $\left.p<.01, \eta_{p}^{2}=0.09\right)$. Contrary to [H3], the score of Ownership with abstract avatars was significantly higher than that with realistic avatars. For Response and Presence, no significant differences were observed. These results did not support [H3].

\section{Correlation Analysis}

To test [H4] and to specify if any of the variables from the questionnaire and SCR data were related to the participants' behaviors, we conducted multiple regression analyses. We used the time that each participant took before they first walked through the wall across rooms (Figure 7 right) as an index to represent the tendency of behaviors throughout the experiment. We also used the VR- and video-game-experiences (both 1-5) as predictors. As a result, only the subjective score of presence was found to be a marginally significant predictor $(p=.06)$. To further test the strength of the relationship between presence and behavior indices, polyserial correlation analyses, which are used for the data between a quantitative and an ordinal variable, were conducted. The results revealed a significant weak correlation $(\rho=0.23, p<.05)$. Specifically, the participants who took a shorter time before walking through the wall tended to have lower sense of presence, supporting [H4].

\section{DISCUSSION}

The main findings are as follows:

1) The results consistently showed that participants who had the full-body human avatar tended to refrain from walking through the walls, as evident from the ratio in room $2(p<.05$ for detour), time in room 3 ( $p<.05$ against $\mathrm{H}$ and $\mathrm{R}$ ), and time across rooms ( $p<.01$ against $\mathrm{H}$ and $p<.05$ against $\mathrm{R}$ ), which supported [H1] and [H2]. In addition, participants with the Human Hand avatar showed tendencies to walk through the walls, with fewer indices, as seen from the ratio in room 2 ( $p<.05$ for walking through and $p=.07$ for detour) and time in room 4 ( $p=.06$ against $C)$.

2) The SCR for full-body-threat (i.e., walking through the lightning wall) in the full-body conditions ( $\mathrm{R}$ or F) showed a trend of being greater than that in the hand-only conditions $(\mathrm{C}$ or $\mathrm{H})(p=.08)$. For the hand-threat (i.e., touch the threatening stimuli), there were no significant differences among the avatar conditions. In addition, the subjective ratings of ownership of the participants embodied with abstract avatars ( $\mathrm{C}$ or $\mathrm{R}$ ) were significantly higher than that of those with realistic avatars ( $\mathrm{H}$ or $\mathrm{F})(p<.01)$. In contrast, the ratings of presence and response to threat were not significantly different among the avatar conditions. These results did not support [H3].

3) The participants with a lower sense of presence tended to take less time before walking through the walls $(p<.05)$, which supported [H4]. Their behavioral tendency was explained only by presence instead of other indices (ownership, response, SCR, VR-experience, and video-game-experience).

Overall, the results showed that the embodiment of highvisibility and high-anthropomorphism self-avatars could discourage participants from walking through the walls, presumably because of a higher sense of presence. More generally, we demonstrated that a higher visual fidelity of self-avatars can facilitate realistic user behavior in the VE. The major difference 
between our results and those of previous studies is that most of them found no significant effect of the presentation [1], anthropomorphism [8], or visibility [33] of self-avatars on the game performance [33], affordance judgment [1,7], and behavior [1]. The only exceptional study, by Lin et al. [31], showed that the presentation of self-avatars had an effect on affordance judgment and the corresponding behavior. However, they did not investigate either the anthropomorphism factor or the visibility factor. Moreover, it is also interesting to note that when the participants were asked in the post-experiment interview about why they hesitated or decided to pass through the wall, none of them mentioned their avatars. This shows that the participants' actions were affected by their avatar designs, even though they were not conscious of this fact. Our finding is important because our results suggest that simply by changing the visibility and the level of anthropomorphism, a VE designer can implicitly navigate users to behave realistically (i.e., avoiding the wall) or unrealistically (i.e., passing through the wall) depending on the situation.

The main concern of our study, i.e., the hypothesis that selfavatars of higher fidelity would discourage the users from walking through the virtual walls without any explicit feedback, was supported by the experiment. Nevertheless, there are some unexpected results that need further discussions. First, unlike behavioral data, we did not observe any significant differences between the subjective scores of presence and SCR among self-avatar groups. This raises the concern that the selfavatar affected the behavior irrespective of the level of sense of presence. However, the result of the correlation analysis indicates that the change in behavior could be explained by the sense of presence. In addition, the SCR data was noisy and not much reliable because of the practical constraints that it was recorded during the participants' active movements. Hence, we consider that in our study the behavioral data could reflect the presence more apparently than the questionnaire and the physiological data. Nevertheless, these contradictory results may imply that there might be other factors than presence that affect the relation between self-avatar appearance and users' behaviors in VEs. For example, the semantic aspects of self-avatars, e.g., skin color [43] and attractiveness [68], have also been shown to affect users' attitude and behavior through stereotype or memory, which is automatically associated with avatars (i.e., Proteus effect). Although in this study we focused on visual fidelity of self-avatars, the semantics of each distinctive avatar could also influence the behavior. Therefore, further research is necessary to clarify the mechanism.

Second, contrary to expectation, the results of the questionnaire indicated that the abstract self-avatars evoked stronger SoBO compared with the realistic self-avatars. One possible reason can be the uncanny valley effect [39]. Previous studies [34, 29] have revealed that robotic and cartoon-like full-body avatars elicit a slightly stronger SoBO than realistic human avatars. However, in the case of hand-only avatars, a number of studies have shown that realistic human virtual hands induced greater SoBO compared with various virtual objects (e.g., sphere, board, block, and arrow) [69, 2, 30, 42]. One noticeable difference between existing studies and our study is that, even though the existing studies showed arbitrary virtual objects to represent participants' hands, we showed virtual controllers with high visual realism that moved in nearly complete correspondence with the physical controller. In addition, Human Hand avatars were displayed as having open palms, even though the participants closed their fists to hold the controllers. Hence, compared with Human Hand avatars, it is possible that Controller avatars produced higher visuomotor and visuo-proprioceptive synchrony, which is a critical factor for inducing SoBO [27]. It was also remarkable that visibility did not affect SoBO. To the best of our knowledge, this is the first study that compares full-body and hand-only avatars with regard to SoBO and presence. Interestingly, the SCR results revealed a marginally significant difference in visibility for the full-body threat. Hence, it still remains unclear if the hand-only avatars elicited an equivalent level of the full-body ownership illusion, compared with full-body avatars.

Similarly, with some indices, participants with the Human Hand avatar were likely to walk through the walls, although participants with the Controller avatar did not show such a tendency. This may be also because the participants perceived higher SoBO when they saw the Controller avatar rather than the Human Hand avatar. However, we need to conduct further studies with various avatar designs to clarify the actual reason.

Considering our results that the effect of self-avatar appearance on behavior is presumably mediated by the sense of presence, improving presence by using other factors may also contribute to preventing users from passing through the walls, such as passive haptics [24], visual realism of VEs [52], and avatar personalization [65]. In addition, combining other approaches to prevent penetration, such as multisensory feedback from wall collisions, would further improve the effectiveness. Combinations and comparisons with these parameters and approaches constitute prospects for future studies. Moreover, the results also indicate that high-fidelity self-avatars can potentially improve a wide variety of VR applications where realistic user responses are required in VEs, e.g., to seriously engage users in evacuation drills, and to the intensity of exposure therapy for PTSD depending on stages. Nevertheless, generalisability of the results for practical applications is subject to certain limitations because the scope of this study is limited to the issue of users' walk-through-wall behaviors. For instance, the effect of self-avatar visibility may not be applicable to behaviors unrelated to the body penetration.

\section{CONCLUSION}

To test our hypothesis that self-avatars with high visual fidelity would induce users to refrain from passing through virtual walls, we investigated whether the anthropomorphism and visibility of self-avatars could influence participants' behavior with respect to walls in room-scale VEs. The results of the experiment showed that the use of a full-body realistic avatar discouraged participants from walking through the walls, presumably because of a higher sense of presence.

\section{ACKNOWLEDGEMENTS}

The authors thank Yuki Zenimoto and Akira Kitasaka for their help in conducting the experiment. The study was partially supported by JST PRESTO (JPMJPR17J6). 


\section{REFERENCES}

[1] Abdulaziz Alshaer, Holger Regenbrecht, and David O'Hare. 2017. Immersion factors affecting perception and behaviour in a virtual reality power wheelchair simulator. Applied Ergonomics 58 (2017), 1-12. DOI : http://dx.doi.org/10.1016/j. apergo.2016.05.003

[2] Ferran Argelaguet, Ludovic Hoyet, Michaël Trico, and Anatole Lécuyer. 2016. The role of interaction in virtual embodiment: Effects of the virtual hand representation. In Proceedings of the IEEE Conference on Virtual Reality (IEEE VR '16). 3-10. DOI : http://dx.doi.org/10.1109/VR.2016.7504682

[3] K Carrie Armel and Vilayanur Subramanian Ramachandran. 2003. Projecting sensations to external objects: evidence from skin conductance response. Proceedings. Biological sciences / The Royal Society 270, 1523 (2003), 1499-1506. DOI: http://dx.doi.org/10.1098/rspb.2003.2364

[4] Mahdi Azmandian, Mark Hancock, Hrvoje Benko, Eyal Ofek, and Andrew D Wilson. 2016. Haptic Retargeting: Dynamic Repurposing of Passive Haptics for Enhanced Virtual Reality Experiences. In Proceedings of the SIGCHI Conference on Human Factors in Computing Systems (CHI '16). 1968-1979. DOI :

http://dx.doi.org/10.1145/2858036.2858226

[5] Jeremy N. Bailenson, Nick Yee, Dan Merget, and Ralph Schroeder. 2006. The effect of behavioral realism and form realism of real-time avatar faces on verbal disclosure, nonverbal disclosure, emotion recognition, and copresence in dyadic interaction. Presence: Teleoperators and Virtual Environments 15, 4 (2006), 359-372. DOI :

http://dx.doi.org/10.1162/pres.15.4.359

[6] Frank Biocca. 1997. The Cyborg's Dilemma : Progressive Embodiment in Virtual Environments Minding the Body, the Primordial Communication Medium. Journal of computer-mediated communication 3, 2 (1997), 1-29. DOI :

http://dx.doi .org/10.1111/j.1083-6101 . 1997.tb00070.x

[7] Bobby Bodenheimer, Sarah Creem-Regehr, Jeanine Stefanucci, Elena Shemetova, and William B. Thompson. 2017. Prism aftereffects for throwing with a self-avatar in an immersive virtual environment. In Proceedings of the IEEE Conference on Virtual Reality (IEEE VR'17). 141-147. DOI :

http://dx.doi.org/10.1109/VR.2017.7892241

[8] Bobby Bodenheimer and Qiang Fu. 2015. The effect of avatar model in stepping off a ledge in an immersive virtual environment. In Proceedings of the ACM SIGGRAPH Symposium on Applied Perception (SAP '15). ACM Press, 115-118. DOI :

http://dx.doi.org/10.1145/2804408.2804426

[9] Mette Boldt, Michael Bonfert, Inga Lehne, Melina Cahnbley, Kim Korschinq, Loannis Bikas, Stefan Finke, Martin Hanci, Valentin Kraft, Boxuan Liu, Tram Nguyen, Alina Panova, Ramneek Singh, Alexander
Steenbergen, Rainer Malaka, and Jan Jan Smeddinck. 2018. You Shall Not Pass: Non-Intrusive Feedback for Virtual Walls in VR Environments with Room-Scale Mapping. In Proceedings of the IEEE Conference on Virtual Reality (IEEE VR '18). 143-150. DOI : http://dx.doi.org/10.1109/VR.2018.8446177

[10] JJ Jason J Braithwaite, Dr Derrick, G Watson, Robert Jones, Mickey Rowe, DG Watson, Jones Robert, and Rowe Mickey. 2013. A Guide for Analysing Electrodermal Activity (EDA) \& Skin Conductance Responses (SCRs) for Psychological Experiments.... (2013), 1-42. DOI :

http://dx.doi.org/10.1017.S0142716405050034

[11] Eric Burns, Abigail T Panter, Mary C Whitton, Matthew R Mccallus, and Frederick P Brooks. 2006. The Hand Is More Easily Fooled than the Eye: Users Are More Sensitive to Visual Interpenetration than to Visual- Proprioceptive Discrepancy Abstract. Presence 15, 1 (2006), 1-15. DOI :

http://dx.doi.org/10.1162/pres.2006.15.1.1

[12] Lung-Pan Cheng, Eyal Ofek, Christian Holz, Hrvoje Benko, and Andrew Wilson. 2017. Sparse Haptic Proxy: Touch Feedback in Virtual Environments Using a General Passive Prop. In Proceedings of the SIGCHI Conference on Human Factors in Computing Systems (CHI'17). 3718-3728. DOI :

http://dx.doi.org/10.1145/3025453.3025753

[13] Lung-Pan Cheng, Thijs Roumen, Hannes Rantzsch, Sven Köhler, Patrick Schmidt, Robert Kovacs, Johannes Jasper, Jonas Kemper, and Patrick Baudisch. 2015. TurkDeck: Physical Virtual Reality Based on People. In Proceedings of the ACM Symposium on User Interface Software and Technology (UIST '15). 417-426. DOI : http://dx.doi.org/10.1145/2807442.2807463

[14] Catherine Dillon, Ed Keogh, Jonathan Freeman, and Jules Davidoff. 2000. Aroused and Immersed: The Psychophysiology of Presence. In The 3rd Annual International Presence Workshop.

[15] Elham Ebrahimi, Leah S. Hartman, Andrew Robb, Christopher C. Pagano, and Sabarish V. Babu. 2018. Investigating the Effects of Anthropomorphic Fidelity of Self-Avatars on Near Field Depth Perception in Immersive Virtual Environments. In Proceedings of the IEEE Conference on Virtual Reality (IEEE VR '18). 1-8. DOI : http://dx.doi.org/10.1109/VR.2018.8446539

[16] H. Henrik Ehrsson. 2007. The experimental induction of out-of-body experiences. Science 317, 5841 (2007), 1048. DOI : http://dx. doi .org/10.1126/science. 1142175

[17] Jonathan Freeman, S. E. Avons, Ray Meddis, Don E. Pearson, and Wijnand Ijsselsteijn. 2000. Using behavioral realism to estimate presence: A study of the utility of postural responses to motion stimuli. Presence: Teleoperators and Virtual Environments 9, 2 (2000), 149-164. DOI : http://dx.doi.org/10.1162/105474600566691 
[18] Mar Gonzalez-Franco and Tabitha C Peck. 2018. Avatar Embodiment. Towards a Standardized Questionnaire. Frontiers in Robotics and AI 5 (2018), 74. DOI: http://dx.doi.org/10.3389/frobt.2018.00074

[19] Mar Gonzalez-Franco, Tabitha C. Peck, Antoni Rodríguez-Fornells, and Mel Slater. 2014. A threat to a virtual hand elicits motor cortex activation. Experimental Brain Research 232, 3 (2014), 875-887. DOI : http://dx.doi .org/10.1007/s00221-013-3800-1

[20] Mar Gonzalez-Franco, Daniel Pérez-Marcos, Bernhard Spanlang, and Mel Slater. 2010. The contribution of real-time mirror reflections of motor actions on virtual body ownership in an immersive virtual environment. In Proceedings of the IEEE Conference on Virtual Reality (IEEE VR'10). 111-114. DOI: http://dx.doi.org/10.1109/VR.2010.5444805

[21] Xiaochi Gu, Yifei Zhang, Weize Sun, Yuanzhe Bian, Dao Zhou, and Per Ola Kristensson. 2016. Dexmo: An inexpensive and lightweight mechanical exoskeleton for motion capture and force feedback in VR. In Proceedings of the SIGCHI Conference on Human Factors in Computing Systems (CHI '16). 1991-1995. DOI : http://dx.doi .org/10.1145/2858036.2858487

[22] Richard M. Held and Nathaniel I. Durlach. 1992. Telepresence. Presence: Teleoperators and Virtual Environments 1, 1 (1992), 109-112. DOI: http://dx.doi.org/10.1162/pres.1992.1.1.109

[23] Heike Hofmann, Hadley Wickham, and Karen Kafadar. 2017. Letter-Value Plots: Boxplots for Large Data. Journal of Computational and Graphical Statistics 26, 3 (2017), 469-477. DOI :

http://dx.doi.org/10.1080/10618600.2017.1305277

[24] B.E. Insko. 2001. Passive haptics significantly enhances virtual environments. Computer (2001), 100.

[25] Sungchul Jung, Gerd Bruder, Pamela J. Wisniewski, Christian Sandor, and Charles E. Hughes. 2018. Over My Hand: Using a Personalized Hand in VR to Improve Object Size Estimation, Body Ownership, and Presence. In Proceedings of the ACM Symposium on Spatial User Interaction (SUI'18). 60-68. DOI : http://dx.doi.org/10.1145/3267782.3267920

[26] Konstantina Kilteni, Raphaela Groten, and Mel Slater. 2012. The Sense of Embodiment in Virtual Reality. Presence: Teleoperators and Virtual Environments 21, 4 (2012), 373-387. DOI :

http://dx.doi.org/10.1162/PRES_a_00124

[27] Konstantina Kilteni, Antonella Maselli, Konrad P Kording, and Mel Slater. 2015. Over my fake body: body ownership illusions for studying the multisensory basis of own-body perception. Frontiers in human neuroscience 9 (2015), 141. DOI :

http://dx.doi.org/10.3389/fnhum. 2015.00141

[28] Konstantina Kilteni, Jean Marie Normand, Maria V. Sanchez-Vives, and Mel Slater. 2012. Extending body space in immersive virtual reality: A very long arm illusion. PLoS ONE 7, 7 (2012), e40867. DOI: http://dx.doi .org/10.1371/journal . pone. 0040867
[29] Marc Erich Latoschik, Daniel Roth, Dominik Gall, Jascha Achenbach, Thomas Waltemate, and Mario Botsch. 2017. The effect of avatar realism in immersive social virtual realities. In Proceedings of the ACM Symposium on Virtual Reality Software and Technology (VRST'17). 1-10. DOI : http://dx.doi.org/10.1145/3139131.3139156

[30] Lorraine Lin and Sophie Jörg. 2016. Need a hand? How Appearance Affects the Virtual Hand Illusion. In Proceedings of the ACM Symposium on Applied Perception. (SAP '16). 69-76. DOI : http://dx.doi.org/10.1145/2931002.2931006

[31] Qiufeng Lin, John Rieser, and Bobby Bodenheimer. 2015. Affordance judgments in HMD-based virtual environments: Stepping over a pole and stepping off a ledge. ACM Transactions on Applied Perception 12, 2 (2015), 1-21. DOI: http://dx.doi.org/10.1145/2720020

[32] Pedro Lopes, Sijing You, Lung Pan Cheng, Sebastian Marwecki, and Patrick Baudisch. 2017. Providing haptics to walls \& heavy objects in virtual reality by means of electrical muscle stimulation. In Proceedings of the SIGCHI Conference on Human Factors in Computing Systems (CHI '17). 1471-1482. DOI : http://dx.doi.org/10.1145/3025453.3025600

[33] Jean Luc Lugrin, Maximilian Ertl, Philipp Krop, Richard Klupfel, Sebastian Stierstorfer, Bianka Weisz, Maximilian Ruck, Johann Schmitt, Nina Schmidt, and Marc Erich Latoschik. 2018. Any 'Body' There? Avatar Visibility Effects in a Virtual Reality Game. In Proceedings of the IEEE Conference on Virtual Reality (IEEE VR'18). 17-24. DOI :

http://dx.doi.org/10.1109/VR. 2018.8446229

[34] Jean Luc Lugrin, Johanna Latt, and Marc Erich Latoschik. 2015. Avatar anthropomorphism and illusion of body ownership in VR. In Proceedings of the IEEE Conference on Virtual Reality (IEEE VR '15). 229-230. DOI : http://dx.doi .org/10.1109/VR. 2015.7223379

[35] Ke Ma and Bernhard Hommel. 2015. Body-ownership for actively operated non-corporeal objects. Consciousness and Cognition 36 (2015), 75-86. DOI : http://dx. doi.org/10.1016/j.concog.2015.06.003

[36] Antonella Maselli and Mel Slater. 2013. The building blocks of the full body ownership illusion. Frontiers in Human Neuroscience 7, 83 (2013), 1-15. DOI: http://dx.doi.org/10.3389/fnhum. 2013.00083

[37] Erin A. McManus, Betty J. Mohler, Heinrich H. Bülthoff, Bobby Bodenheimer, Stephan Streuber, and Stephan de la Rosa. 2011. The influence of avatar (self and character) animations on distance estimation, object interaction and locomotion in immersive virtual environments. In Proceedings of the ACM SIGGRAPH Symposium on Applied Perception in Graphics and Visualization. (APGV'11). ACM Press, 37. DOI : http://dx.doi .org/10.1145/2077451.2077458 
[38] Michael Meehan, Brent Insko, Mary Whitton, and Frederick P. (Jr) Brooks. 2002. Physiological Measures of Presence in Virtual Environments. ACM Transactions on Graphics (TOG) 21, 3 (2002), 645-652. DOI : http://dx.doi.org/10.1145/566654.566630

[39] Masahiro Mori. 1970. The Uncanny Valley. Energy 7, 4 (1970), 33-35. DOI : http://dx.doi.org/10.1109/MRA.2012.2192811

[40] Kazuki Nagai, Katsuhito Akahane, Soma Tanoue, and Makoto Sato. 2015. Wearable 6-DoF wrist haptic device "sPIDAR-W". In SIGGRAPH Asia Haptic Media and Contents Design, (SA '15). DOI:

http://dx.doi.org/10.1145/2818384.2818403

[41] Kristine L. Nowak and Frank Biocca. 2003. The Effect of the Agency and Anthropomorphism on users' Sense of Telepresence, Copresence, and Social Presence in Virtual Environments. Presence: Teleoperators and Virtual Environments 12, 5 (2003), 481-494. DOI : http://dx.doi.org/10.1162/105474603322761289

[42] Nami Ogawa, Takuji Narumi, and Michitaka Hirose. 2019. Virtual Hand Realism Affects Object Size Perception in Body-Based Scaling. In Proceedings of the IEEE Conference on Virtual Reality (IEEE VR '19). 519-528. DOI:

http://dx.doi.org/10.1109/VR. 2019.8798040

[43] Tabitha C. Peck, Sofia Seinfeld, Salvatore M. Aglioti, and Mel Slater. 2013. Putting yourself in the skin of a black avatar reduces implicit racial bias. Consciousness and Cognition 22, 3 (2013), 779-787. DOI:

http://dx.doi.org/10.1016/j. concog.2013.04.016

[44] Catherine Preston, Benjamin J. Kuper-Smith, and H. Henrik Ehrsson. 2015. Owning the body in the mirror: The effect of visual perspective and mirror view on the full-body illusion. Scientific Reports 5 (2015), 18345. DOI : http://dx.doi.org/10.1038/srep18345

[45] Jun Rekimoto. 2013. Traxion: A tactile interaction device with virtual force sensation. In Proceedings of the ACM Symposium on User Interface Software and Technology (UIST '13). 427-431. DOI : http://dx.doi .org/10.1145/2501988.2502044

[46] Brian Ries, Victoria Interrante, Michael Kaeding, and Lee Anderson. 2008. The effect of self-embodiment on distance perception in immersive virtual environments. In Proceedings of the ACM Symposium on User Interface Software and Technology (UIST '08). 167. DOI:http://dx.doi .org/10.1145/1450579.1450614

[47] Brian Ries, Victoria Interrante, Michael Kaeding, and Lane Phillips. 2009. Analyzing the effect of a virtual avatar's geometric and motion fidelity on ego-centric spatial perception in immersive virtual environments. In Proceedings of the ACM Symposium on User Interface Software and Technology (UIST '09). 59. DOI: http://dx.doi.org/10.1145/1643928.1643943

[48] Maria V. Sanchez-vives and Mel Slater. 2005. From Presence Towards Consciousness. Nature Reviews Neuroscience 6, 10 (2005), 332. DOI : http://dx.doi.org/10.1038/nrn1651
[49] Valentin Schwind, Pascal Knierim, Cagri Tasci, Patrick Franczak, Nico Haas, and Niels Henze. 2017. "These are not my hands!"; : Effect of Gender on the Perception of Avatar Hands in Virtual Reality. In Proceedings of the SIGCHI Conference on Human Factors in Computing Systems (CHI '17). 1577-1582. DOI: http://dx.doi.org/10.1145/3025453.3025602

[50] Valentin Schwind, Lorraine Lin, Massimiliano Di Luca, Sophie Jörg, and James Hillis. 2018a. Touch with Foreign Hands: The Effect of Virtual Hand Appearance on Visual-Haptic Integration. In Proceedings of the ACM Symposium on Applied Perception. (SAP '18). ACM, 1-8. DOI : http://dx . doi .org/10.1145/3225153.3225158

[51] Valentin Schwind, Sven Mayer, Alexandre Comeau-Vermeersch, Robin Schweigert, and Niels Henze. 2018b. Up to the fingertip: The effect of avatars on mid-air pointing accuracy in virtual reality. In Proceedings of the ACM Symposium on Computer-Human Interaction in Play. (CHI PLAY'18). 489-502. DOI :

http://dx.doi .org/10.1145/3242671.3242675

[52] Mel Slater, Pankaj Khanna, Jesper Mortensen, and Insu Yu. 2009. Visual Realism Enhances Realistic Response in an Immersive Virtual Environment. IEEE Computer Graphics and Applications 29, 3 (2009), 76-84. DOI : http://dx. doi . org/10.1109/MCG.2009.55

[53] Mel Slater and Martin Usoh. 1994. Body centred interaction in immersive virtual environments. Artificial life and virtual reality 1 (1994), 1-22. DOI:

http://dx.doi.org/10.1.1.109.7613

[54] Bernhard Spanlang, Jean-Marie Normand, David Borland, Konstantina Kilteni, Elias Giannopoulos, Pomes Ausias, Mar Gonzalez-Franco, Daniel Perez-Marcos, Jorge Arroyo-Palacios, Xavi Navarro Muncunill, and Mel Slater. 2014. How to Build an Embodiment Lab: Achieving Body Representation Illusions in Virtual Reality. Frontiers in Robotics and AI 1, November (2014), 1-22. DOI:

http://dx.doi.org/10.3389/frobt.2014.00009

[55] Kay Stanney and Gavriel Salvendy. 1998. Aftereffects and sense of presence in virtual environments: Formulation of a research and development agenda. International Journal of Human-Computer Interaction 10, 2 (1998), 135-187.

[56] Anthony Steed, Ye Pan, Fiona Zisch, and William Steptoe. 2016. The impact of a self-avatar on cognitive load in immersive virtual reality. Proceedings - IEEE Virtual Reality 2016-July (2016), 67-76. DOI: http://dx.doi.org/10.1109/VR. 2016.7504689

[57] Frank Steinicke, Gerd Bruder, Jason Jerald, Harald Frenz, and Markus Lappe. 2010. Estimation of detection thresholds for redirected walking techniques. IEEE Transactions on Visualization and Computer Graphics 16, 1 (2010), 17-27. DOI:

http://dx.doi.org/10.1109/TVCG.2009.62 
[58] William Steptoe, Anthony Steed, and Mel Slater. 2013. Human tails: Ownership and control of extended humanoid avatars. IEEE Transactions on Visualization and Computer Graphics 19, 4 (2013). DOI:

http://dx.doi.org/10.1109/TVCG.2013.32

[59] Stephan Streuber, Stephan De La Rosa, Laura Trutoiu, Heinrich H. Bülthoff, and Betty J. Mohler. 2009. Does Brief Exposure to a Self-avatar Affect Common Human Behaviors in Immersive Virtual Environments? In Proceedings of the Conference of the European Association for Computer Graphics (Eurographics '09). 33-36. DOI : http://dx. doi .org/10.2312/egs . 20091042

[60] Gaetano Tieri, Emmanuele Tidoni, Enea Francesco Pavone, and Salvatore Maria Aglioti. 2015a. Body visual discontinuity affects feeling of ownership and skin conductance responses. Scientific Reports 5, 1 (2015), 17139. DOI : http://dx. doi .org/10.1038/srep17139

[61] Gaetano Tieri, Emmanuele Tidoni, Enea Francesco Pavone, and Salvatore Maria Aglioti. 2015b. Mere observation of body discontinuity affects perceived ownership and vicarious agency over a virtual hand. Experimental Brain Research 233, 4 (2015), 1247-1259. DOI : http://dx.doi .org/10.1007/s00221-015-4202-3

[62] Tanh Quang Tran, Hyunju Shin, Wolfgang Stuerzlinger, and Junghyun Han. 2017. Effects of virtual arm representations on interaction in virtual environments. In Proceedings of the ACM Symposium on Virtual Reality Software and Technology (VRST'17). ACM, 1-9. DOI : http://dx.doi.org/10.1145/3139131.3139149

[63] Martin Usoh, Kevin Arthur, Mary C Whitton, Rui Bastos, Anthony Steed, Mel Slater, and Frederick P Brooks. 1999. Walking $>$ Walking-in-Place $>$ Flying, in Virtual Environments. In Proceedings of the conference on Computer graphics and interactive techniques (SIGGRAPH' '99). https://dx.doi.org/10.1145/311535.311589

[64] Martin Usoh, Ernest Catena, Sima Arman, and Mel Slater. 2000. Using presence questionnaires in reality.
Presence: Teleoperators and Virtual Environments 9, 5 (2000), 497-503. D0I :

http://dx.doi.org/10.1162/105474600566989

[65] Thomas Waltemate, Dominik Gall, Daniel Roth, Mario Botsch, and Marc Erich Latoschik. 2018. The Impact of Avatar Personalization and Immersion on Virtual Body Ownership, Presence, and Emotional Response. IEEE Transactions on Visualization and Computer Graphics 24, 4 (2018), 1643-1652. DOI :

http://dx.doi.org/10.1109/TVCG.2018.2794629

[66] Bob Witmer and Paul Kline. 1998. Judging perceived and traversed distance in virtual environments. Presence 7, 2 (1998), 144-167. DOI :

http://dx.doi.org/10.1162/105474698565640

[67] Jacob O. Wobbrock, Leah Findlater, Darren Gergle, and James J. Higgins. 2011. The aligned rank transform for nonparametric factorial analyses using only anova procedures. In Proceedings of the SIGCHI Conference on Human Factors in Computing Systems (CHI '11). 143. DOI : http://dx. doi . org/10.1145/1978942 . 1978963

[68] Nick Yee and Jeremy Bailenson. 2007. The proteus effect: The effect of transformed self-representation on behavior. Human Communication Research 33, 3 (2007), 271-290. DOI : http://dx.doi.org/10.1111/j.1468-2958.2007.00299.x

[69] Ye Yuan and Anthony Steed. 2010. Is the Rubber Hand Illusion Induced by Immersive Virtual Reality?. In Proceedings of the IEEE Conference on Virtual Reality (IEEE VR'10). 95-102.

https/dx.doi.org/10.1109/VR.2010.5444807

[70] Yiwei Zhao and Sean Follmer. 2018. A Functional Optimization Based Approach for Continuous 3D Retargeted Touch of Arbitrary, Complex Boundaries in Haptic Virtual Reality. In Proceedings of the SIGCHI Conference on Human Factors in Computing Systems (CHI'18). 1-12. DOI :

http://dx.doi.org/10.1145/3173574.3174118 